A N N A L E S Annales de Bretagne et des Pays de l'Ouest

\title{
Villageois en Touraine. La société à Chédigny de 1590 à 1914
}

Fabrice Mauclair

\section{(2) OpenEdition \\ 1 Journals}

Édition électronique

URL : https://journals.openedition.org/abpo/6577

DOI : $10.4000 / a b p o .6577$

ISSN : 2108-6443

Éditeur

Presses universitaires de Rennes

\section{Édition imprimée}

Date de publication : 10 décembre 2020

Pagination : 219-222

ISBN : 978-2-7535-8225-5

ISSN : 0399-0826

Référence électronique

Fabrice Mauclair, «Villageois en Touraine. La société à Chédigny de 1590 à 1914 », Annales de Bretagne et des Pays de l'Ouest [En ligne], 127-4 | 2020, mis en ligne le 10 décembre 2020, consulté le 06 janvier 2023. URL : http://journals.openedition.org/abpo/6577 ; DOI : https://doi.org/10.4000/abpo. 6577 
mettre en lambris et peindre toutes colonnes dudit chœur en image de Paradis et couronnement de la béate Vierge comme il paraît encore " (traduction d'Augustin Pic dans Augustin Pic et Georges Provost, dir. Yves Mahyeuc. Rennes en Renaissance, Rennes, PUR, 2010, p. 354-355). Certes, il n'est pas question ici du retable mais le choix de représenter le Couronnement de la Vierge semble significatif à deux titres. D'une part, il renforce la probabilité que le maître-autel ait été dédié à la Vierge (de manière assez inattendue dans une cathédrale sous le vocable de saint Pierre); d'autre part, la scène du Couronnement de la Vierge suggère une forme de continuité par rapport au cycle narratif du retable qui s'achève par l'Assomption. L'évêque dominicain aura ainsi voulu prendre sa part de l'embellissement du chœur de la cathédrale, en prolongement du retable dont l'initiative reviendrait plus vraisemblablement au chapitre, hypothèse que renforce aujourd'hui la qualité exceptionnelle de l'objet. Combien l'on regrette ici que les délibérations capitulaires ne soient conservées qu'à partir de 1526 !

Deux autres indices, valables pour la fin du XVII ${ }^{\mathrm{e}}$ siècle, me semblent confirmer la présence dans le chœur de la cathédrale d'un maître-autel désormais quelque peu archaïque par rapport aux dévotions tridentines. En 1687, un inventaire (Arch. dép. Ille-et-Vilaine, $1 \mathrm{G}$ 261/1) atteste en effet la présence d'une " custode d'argent continuellement suspandue sur le grand autel " (donc une suspension eucharistique) et aussi d'" un tabernacle de bois doré pour servir à exposer le Très Saint Sacrement " : ne peut-on y voir les compléments, perçus comme indispensables à la fin du XVII e siècle, à un maître-autel gothique dépourvu de tabernacle? À cette date, la priorité eucharistique donnée au maître-autel tendait sans doute à faire oublier le caractère marial des scènes représentées (d'autant que la cathédrale s'était dotée en 1635 d'un autre autel à la Vierge, dit "du Vœu ", dans le transept Nord). On s'explique mieux qu'en 1755, l'inventaire des prééminences de la cathédrale l'ait caractérisé sommairement comme " la naissance et la vie du Sauveur ".

En retrouvant sa beauté initiale, le retable anversois de la cathédrale livre ainsi une part de ses mystères. Il confirme la réalité de cette "Renaissance rennaise " d'avant 1532, si peu italienne encore mais pas moins "renaissante " pour autant. Et il avive notre attente du livre de synthèse sur la cathédrale Saint-Pierre, entreprise collective en cours depuis plusieurs années mais dont l'heureux aboutissement, sous la direction de Jean-Yves Andrieux, paraît désormais en vue.

Georges Provost

Alain PAuQuet, Villageois en Touraine. La société à Chédigny de 1590 à 1914, Chemillé-sur-Indrois, Éditions Hugues de Chivré, 2020, 383 p.

Avec cet ouvrage, Alain Pauquet, agrégé d'histoire et docteur ès-lettres, nous offre une très belle étude d'histoire rurale. Si aucun reproche ne peut être fait à l'édition - la présentation et la réalisation du livre sont en effet excellentes -, il faut dire d'emblée que le travail de recherche très fouillé auquel s'est livré l'auteur aurait sans doute davantage trouvé son public s'il avait été proposé et diffusé par un éditeur national (ou universitaire). En effet, contrairement à ce que pourrait laisser supposer son titre, le livre d'Alain Pauquet est bien plus qu'une " classique monographie communale ". L'auteur, spécialiste de l'histoire politique et sociale du pays, est du reste un chercheur chevronné. Ancien élève de Maurice Aguhlon, Alain Pauquet a consacré sa thèse à la société en Berry au milieu du XIX ${ }^{\mathrm{e}}$ siècle, thèse qu'il a soutenue en 1993 à Paris 1 Panthéon-Sorbonne et publiée en 1998 aux éditions 
L'Harmattan. L'auteur a aussi fait paraître en 2014 (toujours chez L'Harmattan) Une histoire de la citoyenneté politique en France. 30 documents d'archives du XVIIt siècle à nos jours.

Avec le présent ouvrage, l'historien de la sociabilité berrichonne au temps de Louis-Philippe et de la Seconde République est donc sorti de sa période de prédilection et de son champ d'investigation habituel. La réussite est pourtant bel et bien au rendez-vous, même si, au départ, l'objectif fixé était un peu ambitieux. À la manière d'Alain Corbin avec Le monde retrouvé de Louis-François Pinagot (Flammarion, 1997), Alain Pauquet s'est en effet penché sur les " destins individuels et familiaux » de parfaits " inconnus " (paysans, artisans, nobles, ecclésiastiques, militaires et bourgeois) appartenant tous, de près ou de loin, à une " société villageoise " située au sud-est de la Touraine, précisément dans l'actuelle commune de Chédigny (Indre-et-Loire). En réalité, au moins un des " personnages " évoqués dans le livre d'Alain Pauquet n'est pas un inconnu. En effet, à la fin du XVIII ${ }^{\mathrm{e}}$ siècle, le village de Chédigny a eu pour seigneur un certain La Fayette! Autre précision importante, l'auteur, à la façon des " enquêtes ethnologiques " menées par d'illustres prédécesseurs (dont Edgard Morin), a choisi de concentrer son regard sur les habitants d'un seul et unique hameau, celui de La Joubardière, soit, durant toute la période étudiée, jamais plus d'une dizaine de bâtiments et environ vingt-cinq habitants au maximum. L'auteur entendait ainsi utiliser un "genre historiographique " bien connu des historiens : la micro-histoire. Au final, malgré les difficultés et les travers de la démarche historique choisie (difficultés et travers que l'auteur aurait sans doute dû exposer brièvement en préambule), les apports du livre d'Alain Pauquet sont nombreux.

L'introduction présente l'objectif principal du livre : " retrouver le monde rural d'autrefois et son évolution en sortant de l'ombre une société locale tout entière, à l'échelle d'une commune, depuis la fin du Xvi ${ }^{\mathrm{e}}$ siècle jusqu'à la veille du premier conflit mondial ", le cadre chronologique choisi (qui comprend, ce n'est pas courant, à la fois l'Ancien Régime et le début de l'époque contemporaine) étant " imposé " par les sources. De fait, en amont, avant 1590, l'étude projetée était difficilement réalisable dans la mesure où les archives disponibles étaient rares. En aval, les règles de communicabilité imposaient de s'arrêter au début du siècle précédent.

Pour mener à bien son travail, l'auteur a eu recours à des matériaux multiples et variés : actes notariés (au nombre de 276, étalés entre le XVII et l'orée du XXe siècle), tables alphabétiques du Contrôle des actes, registres de l'Enregistrement et de la Conservation des hypothèques, registres paroissiaux et d'état civil, archives judiciaires, autant de sources (finalement assez " classiques ") qui ont servi de base à " la reconstitution de biographies et de destins familiaux " - c'est une des grandes richesses et originalités du livre - mais aussi à la description détaillée des conditions des baux agricoles, de la vie et de l'habitat des générations d'individus qui se sont succédé à La Joubardière. Pour comprendre comment le " hameau-témoin " et ses habitants s'inséraient dans le "contexte régional et national ", on oserait dire dans la "grande Histoire ", de nombreuses autres sources ont également été convoquées : titres seigneuriaux, archives ecclésiastiques, délibérations municipales, recensements, cadastre, archives administratives ou politiques, notamment électorales. Enfin, pour mener à bien son " enquête de micro-histoire ", Alain Pauquet a utilisé la Monographie de Chédigny, soit le " mémoire " d'un instituteur local rédigé à la fin du XIX ${ }^{e}$ siècle et primé en 1900 à l'occasion de l'exposition universelle de Paris.

Tout en suivant un fil chronologique, le livre comprend deux types de chapitres, judicieusement associés et organisés. Ainsi, si la plupart (huit sur douze) sont consacrés à la vie sociale du hameau choisi, les autres décrivent " les cadres institutionnels, sociaux, démographiques et culturels plus généraux " dans lesquels 
celui-ci " évoluait à l'échelle des seigneuries et des paroisses sous l'Ancien Régime, et de la commune après $1790 »$. En jouant sur deux échelles, l'auteur offre donc la possibilité au lecteur de suivre et d'observer « le processus par lequel cette société locale s'est insérée peu à peu dans la société française globale ». De fait, dans l'ouvrage proposé par Alain Pauquet, on trouvera à la fois des données et des développements très précis sur les "structures ", le fonctionnement et l'organisation de la " société rurale " étudiée (avec de nombreux passages consacrés à la vie agricole et à la démographie locale) ainsi que de larges ouvertures sur les évolutions politiques et sociales de la société française tout entière.

Au total, le livre, extrêmement clair et bien écrit et, qui plus est, d'une grande richesse par les descriptions, exemples concrets et analyses qu'il contient, permet de répondre à quelques-unes des hypothèses et questions que les historiens du monde rural se posent depuis de nombreuses années. En ce sens, le travail d'Alain Pauquet vient confirmer ou préciser certaines des réponses déjà apportées. Il en est ainsi de la place des femmes dans la "société villageoise " d'autrefois. Longtemps sous-évaluée, celle-ci est désormais appréhendée et décrite à sa plus juste valeur. Les Villageois en Touraine en offre une parfaite illustration. Plus spécifiquement, l'auteur observe à propos des redevances seigneuriales " le non-paiement de plus en plus généralisé " de celles-ci "pendant les deux décennies précédant la Révolution française ". Ce phénomène, suggère l'auteur, aurait pu préparer "l'effondrement " du "régime seigneurial " survenu en 1789. Quant à la fameuse " réaction féodale ", que les historiens situent généralement dans la seconde moitié du $\mathrm{XVIII}^{\mathrm{e}}$ siècle, Alain Pauquet l'observe dans la localité étudiée "déjà au XVII siècle ". Autre spécificité relevée à Chédigny : le XvIII siècle, " qu'on se représente habituellement en proie à une véritable expansion économique à partir de 1730 environ ", apparaît ici " en relative stagnation ", en partie à cause " d'incidents climatiques " locaux. Néanmoins, l'auteur observe qu'au cours du siècle des Lumières, " la situation économique précaire " du village n'a pas empêché, comme partout ailleurs, l'éclosion d'" une sensibilité nouvelle " et " la diffusion des idées qui ont préparé et réalisé la Révolution française ".

Autre démonstration intéressante faite dans le livre : " aux XVII ${ }^{\mathrm{e}}$ et XVIII ${ }^{\mathrm{e}}$ siècles, les métayers ne restaient que quelques années dans la même exploitation ". De même, quels que soient les propriétaires, "les maisons, métairies, borderies [un terme apparu à Chédigny vers les années 1730-1740] et locatures étaient vendues et revendues assez fréquemment ". Ainsi, contrairement à l'idée autrefois couramment admise selon laquelle les familles de paysans restaient naguère dans les mêmes demeures plusieurs générations durant, "c'est au contraire l'image d'un brassage et d'un renouvellement constant qui ressort ". Par ailleurs, en termes d'évolutions sociales, l'auteur note que dans le village étudié, "passer du statut de petit laboureur ou même de journalier à celui de laboureur enrichi semble avoir été au XVII ${ }^{\mathrm{e}}$ siècle plus facile qu'au XVIII ${ }^{\mathrm{e}}$ [...] Quant aux laboureurs métayers ou fermiers qui les employaient, leur sort semble aussi s'être aggravé au XvIII siècle". Ainsi, " pris globalement, le monde paysan local " a subi durant ce siècle " un appauvrissement, voire une dépossession, au profit de la noblesse et de la bourgeoisie des notaires, des officiers et des marchands fermiers". Certes, par la suite, la Révolution française va " commencer à changer ce rapport de force, mais seule la croissance de la production et des échanges au cours des $\mathrm{XIX}^{\mathrm{e}}$ et $\mathrm{XX} \mathrm{x}^{\mathrm{e}}$ siècles permettra de sortir peu à peu de la pauvreté " qui affectait jusque-là le monde paysan. Du reste, à Chédigny comme ailleurs, surtout dans la deuxième moitié du XVII ${ }^{\mathrm{e}}$ siècle et au début du siècle suivant, certains seigneurs ont connu eux aussi " les affres de l'endettement ", tel Joseph de Jussac, disparu mystérieusement (suicide?) au début des années 1670. Un peu plus tard, après la Révolution et l'Empire, l'auteur 
observe que la commune a connu " la domination durable d'une bourgeoisie foncière et rentière caractéristique du XIX siècle ". D'une manière générale, Alain Pauquet décrit comment, au cours du XIX ${ }^{\mathrm{e}}$ siècle, Chédigny est sorti de "l'isolement relatif " dans lequel la commune était plongée depuis des siècles, en particulier à cause des problèmes posés par l'absence de pont sur son territoire. À ce sujet, le dernier chapitre, sans doute l'un des plus réussis du livre, montre de manière extrêmement claire et précise comment le village a effectué, de 1815 à 1914, " son insertion dans la société globale ". Parmi les éléments qui ont contribué à cette " insertion ", l'auteur évoque bien évidemment l'instruction publique, les élections, les aménagements publics, les transports et, moins souvent évoqués, la garde nationale ou encore le " fait culturel ». Du reste, au fil du livre, et de manière souvent très fine et concrète, l'auteur fournit de nombreux éléments permettant d'observer sur le temps long les changements survenus à Chédigny dans le domaine du rapport à l'espace, pour les paysans comme pour les " élites ", et à propos des évolutions relatives aux interactions et contacts avec le "monde extérieur ", le tout s'opérant grâce à une " série de connexions croissantes ".

Voici, parmi d'autres, quelques-unes des observations et conclusions contenues dans le bon livre d'Alain Pauquet, un livre qui devrait être utile aussi bien aux historiens modernistes que contemporainistes, qu'ils s'intéressent à l'histoire rurale, sociale ou encore politique du pays.

Fabrice MAUCLAIR

MaIlLARD, Brigitte et MAILLARD, Jacques, L'intendance de Tours à la fin du XVII siècle (Touraine, Anjou, Maine). Édition critique du mémoire "pour l'instruction du duc de Bourgogne ", Paris, éditions du CTHS, 2019, 774 p.

Dans les débuts du règne personnel de Louis XIV, Jean-Baptiste Colbert, qui était pour l'heure le membre le plus important du Contrôle royal des finances mis en place en septembre 1661, avait conçu un projet d'histoire générale de la France et demandé à " MM les maîtres des requêtes commissaires départis pour l'exécution des ordres du roi ", autrement dit les intendants des provinces, de rechercher les personnes capables de faire ce travail. En 1663, il leur fit parvenir une Instruction leur ordonnant de mener une enquête détaillée sur leurs circonscriptions, les " généralités ", dans le but essentiel de connaître leurs disponibilités financières en vue d'alimenter le Trésor royal. Une trentaine d'années plus tard, en 1697, une autre enquête plus développée fut adressée aux intendants - dont celui de la généralité de Tours Thomas Hue de Miromesnil -, le questionnaire pour l'éducation du duc de Bourgogne, par le duc de Beauvilliers (1648-1714), Paul de Saint-Aignan, le propre gendre de Colbert. Le mémoire qui fut rédigé en réponse a été souvent attribue à Miromesnil lui-même bien qu'il semble n'avoir nullement participé à sa rédaction. Les principaux auteurs ou inspirateurs de ce texte furent plutôt, pour la Touraine Pierre Carreau (un érudit local qui avait longtemps songé à rédiger une histoire de sa province, un projet abandonné à la mort de Colbert) et l'abbé Louis de Courcillon de Dangeau, pour l'Anjou Nicolas Pétrineau sieur des Noulis, président de la prévôté d'Angers), et pour le Maine Julien Leclerc du Flécheray procureur fiscal du comté de Laval dont il avait rédigé une Description qui sera publiée ultérieurement, et là encore Dangeau dont la famille était originaire du Maine.

Bien que 34 copies du mémoire " pour l'instruction du duc de Bourgogne » dans son intégralité soient aujourd'hui disponibles, répartis dans 17 dépôts d'archives, 